\title{
Reconstructed Elzaki Transform Method for Delay Differential Equations with Mamadu-Njoseh Polynomials
}

\author{
E. J. Mamadu and H. I. Ojarikre \\ Department of Mathematics, Delta State University, Abraka 330106, Nigeria
}

\begin{abstract}
One of the solution techniques used for ordinary differential equations, partial and integral equations is the Elzaki Transform. This paper is an extension of Mamadu and Njoseh [1] numerical procedure (Elzaki transform method (ETM)) for computing delay differential equations (DDEs). Here, a reconstructed Elzaki transform method (RETM) is proposed for the solution of DDEs where Mamadu-Njoseh polynomials are applied as basis functions in the approximation of the analytic solution. Using this strategy, a numerical illustration as in Ref.[1] is provided to the RETM as a basis for comparison to guarantee accuracy and consistency of the method. All numerical computations were performed with MAPLE 18 software.
\end{abstract}

Key words: Elzaki transform method, Mamadu-Njoseh polynomials, delay differential equations.

\section{Introduction}

In the past and present, delay differential equations (DDEs) are found in many physical and biological phenomena. They serve as mathematical models for many realistic physical and biological situations such as the spread of contagious diseases, population dynamics, electrodynamics, etc. There exist several mechanisms in solving DDEs. These methods are either analytic or numerical. Many of these analytic methods (such as Laplace transform method, residual control method, method of steps, $\theta$-step method, etc.) are practically difficult to handle without the aid of computer algebra. Even with computer algebra the many transformations either through linearization or weak assumptions render the methods complex and troublesome in implementation.

Numerical procedures offer more comprehensive approach for solving DDEs. They give approximations of the analytic solution of the DDEs explicitly. There exist several numerical schemes [1-3] developed and implemented over the years for solving DDEs. Many of these numerical methods are quite

Corresponding author: Henrietta Ify Ojarikre, research field: operations research. explicit and straightforward exhibiting a great sense of convergence. Investigations into the convergence of DDEs have been on the increase. For instance, Bellen and Zennaro [4] studied the convergence of DDEs with time-dependent delays. In like manner, Refs. [5-8] studied the convergence of DDEs with respect to state-dependent delay. Enright and Hayashi [9] gave an insightful investigation of the convergence of DDEs with neutral and retarded delays. Investigations into the stability of DDEs have not been left out. Drivers [10] studied an extended one-step approach for DDEs with a depth analysis of DDEs in a given region called the p-stability region.

This paper is motivated by the desire to solve DDEs with much ease. To this end, we propose a new numerical procedure called the "Reconstructed Elzaki Transform Method (RETM)". The RETM is a modified version of the standard Elzaki Transform method (ETM) with unique mathematical efficiency. The convergence of solutions of the method is also treated. Numerical illustrations are carried out with the RETM for comparison to ensure accuracy and consistency. All numerical computations have been performed with MAPLE 18 software. ETM has been arranged in Section 2 while RETM is presented Section 
3. The convergence theory and the numerical computations are in Sections 4 and 5 respectively.

\section{Elzaki Transform Method (ETM)}

Let $u(t)$ be analytic in $[0, \infty]$ for $t \geq 0$. The Elzaki transform [11-13] of $u(t)$ defines a function $q$ given as

$$
E[g(t)]=q \int_{0}^{\infty} g(t) e^{-} d t
$$

\subsection{Properties of ETM}

Some relevant properties of ETM essential to this study include;

(1) $E\left[t^{n}\right]=n ! q^{n+2}$

(2) $E^{-1}\left[q^{n+2}\right]=\frac{t^{n}}{n !}$

Proof. (i). By definition,

$$
\begin{gathered}
E[g(t)]=q \int_{0}^{\infty} g(t) e^{-\frac{q}{t}} d t \Rightarrow E\left[t^{n}\right]= \\
q \int_{0}^{\infty} t^{n} e^{-\frac{q}{t}} d t .
\end{gathered}
$$

Using integration by part:

$$
E\left[t^{n}\right]=q\left[\left(\frac{t^{n+2} e^{-\frac{q}{t}}}{q}\right)_{0}^{\infty}-\frac{n}{q} \int_{0}^{\infty} t^{n+1} e^{-\frac{q}{t}} d t\right]
$$

Simplifying further, we have

$$
\begin{aligned}
& E\left[t^{n}\right]=q\left[\left(-\frac{n t^{n+3} e^{-\frac{q}{t}}}{q^{2}}\right)_{0}^{\infty}\right. \\
&\left.+\frac{n(n+1)}{q^{2}} \int_{0}^{\infty} t^{n+2} e^{-\frac{q}{t}} d t\right]
\end{aligned}
$$

since $\left(\frac{t^{n+2} e^{-\frac{q}{t}}}{q}\right)_{0}^{\infty} \rightarrow 0$.

Continuing the above sequence will give

(ii). From (i), we have,

$q^{n+2}=\frac{E\left[t^{n}\right]}{n !} \Rightarrow E^{-1}\left[q^{n+2}\right]=\frac{E^{-1}\left[E\left[t^{n}\right]\right]}{n !}=\frac{t^{n}}{n !}$

\section{Reconstructed Elzaki Transform Method (RETM)}

Consider the generalized DDE of the form

$$
u^{(n)}(t)=g\left(t, u(t), u\left(\alpha_{1}\right), \ldots, u\left(\alpha_{k}\right)+f(t)\right.
$$

with the initial condition

$$
u^{j}(0)=\sum_{i=0}^{n-1} q^{2-n+1} \frac{d^{i} g(0)}{d t^{i}}, j=0,1,2, \ldots
$$

where $\alpha_{j}=\alpha_{j}(t, u(t))$ for $\alpha_{j} \leq t$ are the delay functions, and $f(t)$ is the source term.

Shampine and Thompson [14] proposed a Matlab routine that solves the state and variable DDEs explicitly using the residual error control mechanism. Wazwaz [15] presented an algorithm for handling higher orders of integro-differential equations. His approach is obviously different from our work. However, we intend to follow the footprint of Ref. [1] by proposing a Maple 18 routine through a reconstructed Elzaki transform method (RETM).

The first step in RETM is the construction of initial approximations for Eq. (1.1). At such, let the initial approximation for Eq. (1.1) be given as

$$
u_{0}(t)=E^{-1}\left[q E\left[\sum_{i=0}^{n-1} a_{i} \varphi_{i}(t)+f(t)\right]\right]
$$

where $a_{i}$ 's are unknown constant to be determined, $\varphi_{i}(t), \quad i=0(1)(n-1)$, are the Mamadu-Njoseh polynomials [3] defined in Eq. (1.1), $f(t)$ is the source term, and $n$ denotes the order of the DDE.

It should be noted that Eq. (1.3) satisfies all Elzaki properties given in Section 2.2.1. Suppose that $n=1$ in Eq. (1.3), then

$$
u_{0}(t)=E^{-1}\left[q E\left[a_{0}+f(t)\right]\right]
$$

Using the initial condition $u(0)=q^{2} g(0)$ on Eq. (1.4), we arrive at $a_{0}=q^{2} g(0)$ since $\varphi_{0}(t)=1$. Thus, the initial approximation for first order DDE is given as:

$$
u_{0}(t)=E^{-1}\left[q E\left[q^{2} g(0)+f(t)\right]\right]
$$

For second order DDE, we have that

$$
u_{0}(t)=E^{-1}\left[q E\left[a_{0}+a_{1} t+f(t)\right]\right]
$$

Using the initial condition $u(0)=q^{2} g(0), u^{\prime}(0)=$ $q^{2} g^{\prime}(0)$ on Eq. (1.6), we arrive at 
$a_{0}=q^{2} g(0), a_{1}=q^{2} g^{\prime}(0)$ since $\varphi_{1}(t)=t$. Thus, the initial approximation for second order DDE is given as:

$$
u_{0}(t)=E^{-1}\left[q E\left[q^{2} g(0)+q^{2} g^{\prime}(0) t+f(t)\right]\right]
$$

Similarly, when $n=3$,

$$
\begin{gathered}
u_{0}(t)=E^{-1}\left[q E \left[a_{0}+a_{1} t+\frac{a_{2}}{3}\left(5 t^{2}-2\right)\right.\right. \\
+f(t)]]
\end{gathered}
$$

Using the initial condition $u(0)=q^{2} g(0), u^{\prime(0)}=$ $q^{2} g^{\prime}(0), u^{\prime \prime(0)}=q^{2} g^{\prime \prime}(0)$ on Eq. (1.8), we get

$$
\begin{gathered}
a_{0}=q^{2} g(0)+\frac{1}{5} q^{2} g^{\prime \prime}(0), a_{1}=q^{2} g^{\prime}(0), \\
a_{2}=\frac{3}{10} q^{2} g^{\prime \prime}(0), \text { since } \varphi_{2}(t)=\frac{1}{3}\left(5 t^{2}-2\right) .
\end{gathered}
$$

Thus, the initial approximation for third order DDE is given as:

$$
\begin{aligned}
u_{0}(t)=E^{-1}[q E & {\left[q^{2} g(0)+\frac{1}{5} q^{2} g^{\prime \prime}(0)\right.} \\
& +q^{2} g^{\prime}(0) t \\
& +\frac{1}{10}\left(5 t^{2}-2\right) q^{2} g^{\prime \prime}(0) \\
& +f(t)]]
\end{aligned}
$$

and so on.

We now proceed to derive the RETM for the problem Eq. (1.1) as follows:

Taking the Elzaki transform $E$ on both sides of Eq. (1.1) without the source term,

$$
E\left[u^{(n)}(t)\right]=E\left[g\left(t, u(t), u\left(\alpha_{1}\right), \ldots, u\left(\alpha_{k}\right)\right]\right.
$$

The RETM requires that Eq. (1.10) is rewritten as

$$
E[u(t)]=q E\left[g\left(t, u(t), u\left(\alpha_{1}\right), \ldots, u\left(\alpha_{k}\right)\right]\right.
$$

such that the inverse Elzaki transform on both sides of Eq. (1.11) yields

$$
u(t)=E^{-1}\left[q E\left[g\left(t, u(t), u\left(\alpha_{1}\right), \ldots, u\left(\alpha_{k}\right)\right]\right]\right.
$$

The RETM gives the solution as

$$
u(t)=\sum_{n=0}^{\infty} u_{n}(t)
$$

Thus, Eq. (1.12) becomes:

$$
\begin{aligned}
& \sum_{n=0}^{\infty} u_{n}(t) \\
& =E^{-1}\left[q E\left[\begin{array}{c}
g\left(t, \sum_{n=0}^{\infty} u_{n}(t), \sum_{n=0}^{\infty} u_{n}\left(\alpha_{1}\right),\right. \\
\ldots, \sum_{n=0}^{\infty} u_{n}\left(\alpha_{k}\right)
\end{array}\right]\right.
\end{aligned}
$$

On comparing both sides of Eq. (1.13), we obtain the iterative scheme

$$
\begin{aligned}
& u_{n+1}(t) \\
& =E^{-1}\left[q E\left[g\left(t, u_{n}(t), u_{n}\left(\alpha_{1}\right), \ldots, u_{n}\left(\alpha_{k}\right)\right]\right]\right.
\end{aligned}
$$

Therefore, the computed solution is given as

$$
u(t)=\lim _{N \rightarrow \infty}\left(\sum_{n=0}^{N} u_{n}(t)\right)
$$

For nonlinear DDEs, the nonlinear term is first decomposed as $\sum_{n=0}^{\infty} A n(t)$, where $A_{n}(t)$ 's are Adomion polynomials $[4,5]$, which are determined using the relation

$$
A_{n}(t)=\frac{1}{n !}\left[\frac{d^{n}}{d \alpha^{n}} \widetilde{N}(t)\right]_{\alpha=0}
$$

where $\widetilde{N}(t)=\sum_{i=0}^{n} \alpha^{i} u_{i}(t)$ is a nonlinear operator.

Hence, the iterative scheme Eq. (1.14) becomes,

$$
\begin{aligned}
& u_{n+1}(t) \\
& =E^{-1}\left[q E\left[g\left(t, A_{n}(t), A_{n}\left(\alpha_{1}\right), \ldots, A_{n}\left(\alpha_{k}\right)\right]\right]\right.
\end{aligned}
$$

with the computed solution given as

$$
u(t)=\lim _{N \rightarrow \infty}\left(\sum_{n=0}^{N} u_{n}(t)\right)
$$

\section{Convergence of the Solution}

Theorem. If the solution $u(t)=\sum_{n=0}^{\infty} u_{n}(t)$ is convergent, where $u_{n}(t)$ is defined by Eqs. (1.14) and (1.15), then it must be exact solution of Eq. (1.1).

Proof. Convergence of $\sum_{n=0}^{\infty} u_{n}(t)$ implies $\lim _{n \rightarrow \infty} u_{n}(t)=0$.

Consider the series $\sum_{n=0}^{\infty} u_{n+1}(t)$. Using Eq. (1.16), Eq. (1.14) and $\lim _{N \rightarrow \infty}\left(\sum_{n=0}^{N} u_{n}(t)\right)$ yield 


$$
\begin{gathered}
\sum_{n=0}^{\infty} u_{n+1}(t)=\sum_{n=0}^{\infty}\left(u_{n+1}(t)-u_{n}(t)\right) \\
=\lim _{n \rightarrow \infty} u_{n+1}(t)=\left\{\begin{array}{l}
0, n \leq 1 \\
1, n>1
\end{array}\right.
\end{gathered}
$$

Similarly, using Eq. (1.16), Eq. (1.15) and $\lim _{N \rightarrow \infty}\left(\sum_{n=0}^{N} u_{n}(t)\right)$ yield

$$
\begin{gathered}
\sum_{n=0}^{\infty} u_{n+1}(t)=\sum_{n=0}^{\infty}\left(A_{n+1}(t)-A_{n}(t)\right) \\
=\lim _{n \rightarrow \infty} A_{n+1}(t)=\left\{\begin{array}{l}
0, n \leq 1 \\
1, n>1
\end{array}\right.
\end{gathered}
$$

This completes the proof.

\section{Numerical Illustrations}

In this section, we experiment the RETM on the same examples in Ref. [1] for comparison to ensure accuracy and consistency.

Example 5.1 [1]: Consider the nonlinear DDE

$$
u^{\prime}(t)=1-2 u^{2}(t / 2), t \in[0,1],
$$

with initial condition

$$
u(0)=0 .
$$

The exact solution is $u(t)=\sin t$.

The initial approximation is

$$
u_{0}(t)=E^{-1}\left[q E\left[q^{2} g(0)+f(t)\right]\right] .
$$

Here: $f(t)=1, g(0)=0$.

$$
\Rightarrow u_{0}(t)=E^{-1}[q E[1]]
$$

But $[1]=q^{2}$.

$$
\Rightarrow u_{0}(t)=E^{-1}\left[q^{3}\right]=t .
$$

Since the DDE is nonlinear, we apply the iterative in Eq. (1.15), that is,

$u_{n+1}(t)=E^{-1}\left[q E\left[g\left(t, A_{n}(t), A_{n}\left(\alpha_{1}\right), \ldots, A_{n}\left(\alpha_{k}\right)\right]\right]\right.$

Thus,

$u_{n+1}(t)=-E^{-1}\left[q E\left[2 A_{n}(t / 2)\right]\right], n \geq 0$.

For $n=0: u_{1}(t)=-E^{-1}\left[q E\left[2 A_{0}(t / 2)\right]\right]$

Here,

$$
\begin{aligned}
& A_{0}(t / 2)=t^{2} / 4 \Rightarrow u_{1}(t)=-2 E^{-1}\left[q E\left[t^{2} / 4\right]\right] \\
& =-t^{3} / 3 .
\end{aligned}
$$

For $n=1: u_{2}(t)=-E^{-1}\left[q E\left[2 A_{1}(t / 2)\right]\right]$

Here,

$$
A_{1}(t / 2)=-t^{4} / 48 \Rightarrow u_{2}(t)=\frac{1}{24} E^{-1}\left[q E\left[t^{4}\right]\right]=
$$

$t^{5} / 5$

$$
\begin{aligned}
& \text { For } n=2: u_{3}(t)=-E^{-1}\left[q E\left[2 A_{2}(t / 2)\right]\right] \\
& \text { Here, } A_{2}(t / 2)=t^{6} / 1440 \\
& \Rightarrow u_{3}(t)=2 E^{-1}\left[q E\left[t^{6} / 1440\right]\right]=-t^{7} / 7 !
\end{aligned}
$$

Thus, the approximate solution is given as

$$
\begin{aligned}
u(t)=\lim _{N \rightarrow \infty}\left(\sum_{n=0}^{N} u_{n}(t)\right) & \\
& =t-t^{3} / 3+t^{5} / 5 !-t^{7} / 7 !+\cdots \\
& =\sum_{n=0}^{\infty}\left(-1^{1} n \frac{(t)^{(2 n+1)}}{(2 n+1) !}=\sin t .\right.
\end{aligned}
$$

Example 5.2 [1]: Consider the nonlinear DDE

$$
u^{\prime \prime \prime}(t)=-1+2 u^{2}(t / 2), t \in[0,1],
$$

with initial condition

$$
u(0)=u^{\prime}(0)=u^{\prime \prime \prime}(0)=0 .
$$

The exact solution is $u(t)=-\sin t$.

The initial approximation is

$$
\begin{aligned}
u_{0}(t)=E^{-1}[q E & {\left[q^{2} g(0)+\frac{1}{5} q^{2} g^{\prime \prime}(0)+q^{2} g^{\prime}(0) t\right.} \\
+ & \left.\left.\frac{1}{10}\left(5 t^{2}-2\right) q^{2} g^{\prime \prime}(0)+f(t)\right]\right] .
\end{aligned}
$$

Here: $f(t)=-1, g(0)=g^{\prime}(0)=g^{\prime \prime}(0)=0$.

$$
\Rightarrow u_{0}(t)=-E^{-1}[q E[1]] \text {. }
$$

But $[1]=-q^{2}$.

$$
\Rightarrow u_{0}(t)=-E^{-1}\left[q^{3}\right]=-t \text {. }
$$

Since the DDE is nonlinear, our iterative scheme is given as

$$
\left.u_{n+1}(t)=2 E^{-1}\right)\left[q E\left[A_{n}(t / 2)\right]\right], n \geq 0 .
$$

Hence, we obtain the following iterates: 
For $n=0: u_{1}(t)=t^{3} / 3$

For $n=1: u_{2}(t)=-t^{5} / 5$ !

For $n=2: u_{3}(t)=t^{7} / 7$ !

Thus, the approximate solution is given as

$$
\begin{gathered}
u(t)=\lim _{N \rightarrow \infty}\left(\sum_{n=0}^{N} u_{n}(t)\right) \\
=-\left(t-t^{3} / 3+t^{5} / 5 !-t^{7} / 7 !\right. \\
+\cdots)=-\sin t .
\end{gathered}
$$

\section{Discussion of the Method}

Applying the RETM on the first and third order nonlinear DDEs showed absolute convergence. The computed series solutions converged absolutely to the exact solution for Examples 5.1 and 5.2 respectively. The results obtained agree with those in Ref. [1].

\section{Conclusion}

We have developed and implemented the RETM for solving DDEs. Results obtained showed that the RETM is more feasible DDEs solver than the ETM. It uses less computation time to generate results. Thus, the RETM is accurate and reliable DDEs solver for linear and nonlinear. The work entails some theoretical results including convergence theory. The model presented guarantees stability of solution.

\section{References}

[1] Mamadu, E. J., and Njoseh, I. N. 2017. "Solving Delay Differential Equations by Elzaki Transform Method." Boson Journal of Modern Physics 3 (1): 214-8.

[2] Mohyud-Din, S. T., and Yildirim, A. 2010. "Variational Iteration Method for Delay Differential Equations Using He's Polynomials.” Z. Naturforch 65a: 1045-8.
[3] Mohammed, O. H., and Khlaif, A. 2014. "Adomian Decomposition Method for Solving Delay Differential Equations of Fractional Order." IOSR Journal of Mathematics 10 (6): 1-5.

[4] Ogunfiditimi, F. O. 2015. "Numerical Solution of Delay Differential Equations Using Adomian Decomposition Method." The International Journal of Engineering and Science 4 (5): 18-23.

[5] Bellen, A., and Zennaro, M. 1985. "Numerical Solution of Delay Differential Equations by Uniform Corrections to an Implicit Runge-kutta Method." Numer. Math 47: 301-16.

[6] Ali. 2009. "A Spectral Method for Pantograph-type Delay Differential Equations and Its Convergence Analysis." Journal of Computational Mathematics 27 (2-3): 254-65.

[7] Calvo, M., and Grande, T. 1988. "On the Asymptotic Stability of the $\theta$-methods for Delay Differential Equations." Numer. Math. 54: 257-69.

[8] Driver, R. D. 1977. Ordinary and Delay Differential Equations. New York: Springer-Verlag.

[9] Elzaki, T. M., and Ezaki, S. M. 2011. "On the Elzaki Transform and Ordinary Differential Equation with Variable Coefficients." Advances in Theoretical and Applied Mathematics 6: 41-6.

[10] Ziane, D., and Cherif, M. H. 2015. "Resolution of Nonlinear Partial Differential Equations by Elzaki Decomposition Method." Journal of Approximation Theory and Applied Mathematics 5: 18-30.

[11] Raisinghania, M. D. 2013. Advanced Differential Equations. Sixteenth Revised Edition. New Delhi: Ram Nagar.

[12] Njoseh, I. N., and Mamadu, E. J. 2016. "Numerical Solutions of Fifth Order Boundary Value Problems Using Mamadu-Njoseh Polynomials." Science World Journals 11 (4): 21-4.

[13] Hashim, I. 2006. “Adomian Decomposition Method for Solving BVPs for Fourth Order Integro-differential Equations." Journal of Computational and Applied Mathematics: 658-64.

[14] Shampine, L. F., and Thompson, S. 2001. "Solving DDEs in Matlab." Applied Numerical Mathematics 37 (4): 441-58.

[15] Wazwaz, A. M. 2001. "A Reliable Algorithm for Solving Boundary Value Problems for Higher-order of Integro-differential Equations.” Applied Mathematics and Computation: 327-42. 